\title{
Avaliação Nutricional Em Funcionários De Uma Unidade De Alimentação E Nutrição
}

\section{Nutritional Evaluation In Employees Of A Food And Nutrition Unit}

\author{
Fernanda de Almeida Escobar
}

Palavras-chaves:

Avaliação nutricional

IMC

Hábitos alimentares

Unidade de Alimentação

Nutrição.

\section{Resumo}

A transição nutricional, na qual grande parte da população mundial vive, caracteriza-se pela redução na prevalência de desnutrição com aumento generalizado na prevalência de sobrepeso. Os hábitos alimentares exercem grande influência sobre o crescimento, desenvolvimento e saúde geral dos indivíduos. O índice de massa corporal, criado por Quételet, é um dos critérios mais utilizados atualmente para calcular a prevalência de obesidade, porem, tem suas limitações. O trabalhador de uma UAN sofre a pressão temporal da produção, a qual necessita ajustar-se aos horários de distribuição das refeições, condicionando elou modificando constantemente o modo operatório dos operadores, a fim de atender a demanda. O presente estudo foi desenvolvido para correlacionar avaliação nutricional antropométrica com maus hábitos alimentares em uma UAN e suas consequencias. Foram avaliados 51 trabalhadores, sendo 24 mulheres com idade de 20 a 60 anos, e 27 homens com idade de 19 a 58 anos. 50\% das mulheres e 44,44\% de homens apresentaram IMC eutrofico, 37,5\% das mulheres e 70,37\% dos homens relataram ter boa alimentação. As mulheres mostraram-se mais cientes ao que diz respeito a uma boa alimentação.

\begin{abstract}
The nutritional transition, faced by a great part of the world population, is characterized by the reduction in the malnutrition prevalence with the general increase in the overweight prevalence. Eating habits have a great influence over people's growth, development and health in general. The body mass index (BMI), created by Quételet, is one of the most currently used criteria to measure the overweight prevalence; however, it has limitations. The employee of a Food and Nutrition Unit is under time pressure of production, which has to be adapted to mealtimes, conditioning and/or constantly changing all the operation in order to meet the needs. This work aims to correlate anthropometric nutritional evaluation with bad eating habits in a Food and Nutrition Unit and its consequences. 51 employees were evaluated, 24 women between 20 and 60 years old and 27 men between 19 and 58 years old. $50 \%$ of the women and $44,44 \%$ of the men had eutrofic BMI, $37,5 \%$ of the women and $70,37 \%$ of the men said to have good eating habits. Women seemed more aware of what good eating habits are.
\end{abstract}

\section{Introdução}

Atransição nutricional, na qual grande parte da população mundial vive, caracterizase pela redução na prevalência de desnutrição energética proteica com aumento generalizado na prevalência de sobrepeso (CASTRO, 2004). Isso tem sido atribuído, em parte, a mudanças nos padrões de alimentação, evidentes
Key words:

Nutritional evaluation

$B M I$

eating habits

Food and Nutrition

Unit.

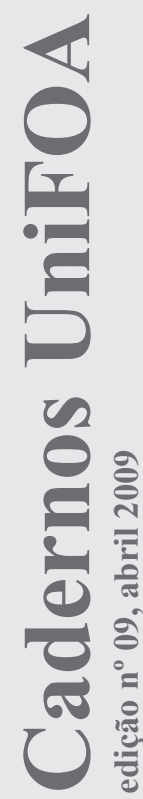

Artigo

Original

Original

Paper 
ricas em fibra protegem contra obesidade, doenças cardiovasculares, diabetes e alguns tipos de câncer. Além disso, estudos clínicos e epidemiológicos sugerem que a gordura dietética tem importante papel no desenvolvimento de doenças cardiovasculares. Apesar dessas evidências, o consumo de dietas ricas em gordura e pobres em frutas, vegetais e cereais integrais ainda é elevado tanto em países desenvolvidos quanto em países em desenvolvimento. Os comportamentos alimentares, associados com diminuição dos níveis populacionais de gasto energético, são consistentes com a importância crescente de doenças crônicas não transmissíveis no perfil de morbimortalidade e com o aumento contínuo da prevalência de obesidade no País. O perfil alimentar da população está fortemente associado a aspectos culturais, socioeconômicos e demográficos, tornando-se necessário uma melhor compreensão desses aspectos no entendimento do comportamento alimentar (NEUTZLING, 2007).

$\mathrm{O}$ índice de massa corporal (IMC) expresso pela relação entre o peso (quilogramas) e a estatura (metros) ao quadrado criado por Quételet (ANJOS,1992), é um dos critérios mais utilizados atualmente para calcular a prevalência de obesidade. Para isso, necessita-se de informações sobre o peso e a altura de cada indivíduo. Tais informações devem ser obtidas, preferencialmente, com base na mensuração direta dos indivíduos, utilizando-se para isso balanças e estadiômetros (SILVEIRA, 2005). O IMC parece ter bom potencial como indicador do estado nutricional em sistemas de vigilância nutricional, já que os dados de peso e altura são (ou pelo menos deveriam ser) rotineiramente coletados durante consultas de adultos, porém, Garn e col. (1986) enumeraram três limitações para o uso do IMC: a correlação com a estatura (que apesar de baixa ainda é significativa), com a massa livre de gordura (principalmente nos homens) e com a proporcionalidade corporal (relação tamanho das pernas/tronco), o que, segundo os autores, poriam em risco a utilização do IMC como indicador de gordura corporal (ANJOS, 1992).

O mercado da alimentação é dividido em alimentação comercial e alimentação coletiva, sendo que os estabelecimentos que trabalham com produção e distribuição de alimentação para coletividades, atualmente, recebem o nome de Unidade de Alimentação e Nutrição (UAN) (PROENÇA, 1997). A produção de refeições para uma clientela definida envolve fatores como: o número de operadores, tipo de alimento utilizado, técnicas de preparo e infraestrutura, exigindo ainda, uma série de equipamentos e utensílios que visam otimizar as operações, tornando-as mais rápidas e confiáveis do ponto de vista da conformidade do produto final (PROENÇA, 1996).

Destaca-se, porém, considerando o arsenal tecnológico disponível para o setor, que a qualidade das refeições está diretamente ligada ao desempenho da mão de obra. Vários autores ressaltam a relação entre a qualidade do trabalho e a qualidade do produto final, salientando-se as preocupações apresentadas em diversos países europeus e EUA para o melhoramento das condições de processamento de refeições (PROENÇA, 1996 e 1997). No Brasil, frequentemente, a produção de refeições exige dos operadores alta produtividade em tempo limitado, porém, em condições inadequadas de trabalho, com problemas de ambiente, equipamento $\mathrm{e}$ processos. Tais condições acabam levando a insatisfações, cansaço excessivo, quedas de produtividade, problemas de saúde e acidentes de trabalho (SANT'ANA, 1994). Dessa forma, o trabalho em UAN tem sido caracterizado por movimentos repetitivos, levantamento de peso excessivo e permanência por períodos prolongados na postura em pé. Além disso, sofre a pressão temporal da produção, a qual necessita ajustar-se aos horários de distribuição das refeições, condicionando e/ou modificando constantemente o modo operatório dos operadores, a fim de atender a demanda (MONTEIRO, 1997).

O presente estudo foi desenvolvido para correlacionar avaliação nutricional antropométrica com maus hábitos alimentares em uma Unidade de Alimentação e Nutrição e suas consequências. Informando aos funcionários a importância de uma alimentação equilibrada, dentro dos padrões nutricionais, e de se ter bons hábitos de vida.

\section{Materiais e Métodos}

A unidade foi escolhida por conveniência, pois o autor prestava estágio na unidade. $O$ objetivo do estudo, seus 
procedimentos e a participação voluntária foram devidamente explicados a todos os 101 funcionários ativos da empresa. Desse total, optou-se por estudar somente os 51 trabalhadores (50,5\%), ocupando as funções nas áreas de produção da padaria, restaurante, confeitaria, setor de compras, escritório, cozinha, serviços gerais, setor de nutrição e garçom. Sendo 24 do sexo feminino com idade de 20 a 60 anos, e 27 do sexo masculino com idade de 19 a 58 anos. Os funcionários foram escolhidos aleatoriamente e analisados separadamente, para identificar cada funcionário utilizou-se um questionário contendo: nome; idade; estado civil; sexo; área onde trabalha. No questionário também continha perguntas fechadas, referentes a hábitos prejudiciais à saúde com opção de responder apenas sim, não ou esporadicamente: Fuma? Consome bebidas alcoólicas? Pratica atividade física? Perguntas fechadas referentes ao hábito alimentar: Consome legumes e verduras nas refeições realizadas na empresa? Na sua opinião, sua alimentação é saudável? E procurou-se saber se os funcionários continham alguma alteração no organismo. Todos os dados foram separados e analisados de acordo com o sexo.

Para realizar a avaliação do estado nutricional, pesou-se e mediu-se a altura utilizando uma balança digital e um estadiômetro, para verificar o IMC de cada um, usando os pontos de corte proposto pela WHO (1997). (Tabela 1)

\begin{tabular}{|l|c|}
\hline \multicolumn{1}{|c|}{ Classificação } & IMC $\left(\mathrm{Kg} / \mathbf{m}^{\mathbf{2}}\right)$ \\
\hline Magreza Grau III & $<16$ \\
\hline Magreza Grau II & $16-16,9$ \\
\hline Magreza Grau I & $17-18,4$ \\
\hline Eutrofia & $18,5-24,9$ \\
\hline Pré-obeso & $25-29,9$ \\
\hline Obesidade Grau I & $30-34,9$ \\
\hline Obesidade Grau II & $35-39,9$ \\
\hline Obesidade Grau III & $\geq 40$ \\
\hline Fonte: WHO (1997) \\
Tabela 1- Niveis de corte para classificação do estado nutricional \\
de adultos de acordo com o indice de massa corporal (IMC)
\end{tabular}

Após a analise do estado nutricional dos funcionários, foi entregue a eles um panfleto informativo sobre alimentação adequada, dando a eles uma noção básica de como se deve fazer para se ter uma boa alimentação e a importância de cada macronutriente (Anexo 1).

\section{Resultados}

Avaliando IMC entre as mulheres foi possível observar que, 12 delas (50\%) encontravam-se eutroficas (Tabela 2), já entre os homens, apenas 12 deles $(44,44 \%)$ apresentaram eutrofia, como se pode observar na Tabela 3, se somarmos os resultados, 15 deles $(55,56 \%)$, a maioria, encontra-se acima do peço ideal. Tanto entre os homens quanto entre as mulheres o restante apresentou-se acima do peço ideal. Com exceção de uma mulher que apresentou magreza grau II.

\begin{tabular}{|c|c|c|}
\hline Classificarăo & $\mathrm{N}^{0}$ de pessoas & Percentual \\
\hline$\overline{\text { Magreza Grau III }}$ & 00 & $0 \%$ \\
\hline Magreza Grau II & 01 & $4,17 \%$ \\
\hline Magreza GrauI & 00 & $0 \%$ \\
\hline Eutrofia & 12 & $50,00 \%$ \\
\hline Pré-obeso & 07 & $29,16 \%$ \\
\hline Obesidade Grau I & 03 & $12,50 \%$ \\
\hline Obesidade Grau II & 00 & $0 \%$ \\
\hline Obesidade Grau III & 01 & $4,17 \%$ \\
\hline \multicolumn{3}{|c|}{ Tabela 2 - Avalią̧ăo nutricional de acordo com o Índice de Massa Corporal (IMC) Femininino } \\
\hline Classificaąäo & $\mathrm{N}^{0}$ de pessoas & Percentual \\
\hline Magreza Grau III & 00 & $0 \%$ \\
\hline & 00 & $0 \%$ \\
\hline Magreza Grau I & 00 & $0 \%$ \\
\hline Eutrofia & 12 & $44,44 \%$ \\
\hline Pré-obeso & 10 & $37,04 \%$ \\
\hline Obesidade Grau I & 4 & $14,81 \%$ \\
\hline $\begin{array}{l}\text { Obesidade Graul II } \\
\text { Opesidade tranIIt }\end{array}$ & 00 & $0 \%$ \\
\hline
\end{tabular}

Quando se perguntou a eles se apresentavam algum tipo de alteração no organismo, obteve-se um bom resultado, $70,82 \%$ das mulheres e $77,79 \%$ dos homens relataram não apresentar alterações em seu organismo.(Tabela 4) Mas, contudo, não foi apresentado por nenhum funcionário qualquer exame laboratorial que comprove ou não a existência de alterações no organismo.

\begin{tabular}{|c|c|c|}
\hline Alteração do organismo & $\begin{array}{c}\text { Percentual } \\
\text { Feminino }\end{array}$ & Percentual Masculino \\
\hline Não apresenta alteraçōes & $70,82 \%$ & $77,79 \%$ \\
\hline Hipotensäo & $12,50 \%$ & $3,70 \%$ \\
\hline Colesterol Elevado & $0 \%$ & $3,70 \%$ \\
\hline Doencas Cardiacas & $4,17 \%$ & $0 \%$ \\
\hline Hipertensão e Triglicerídeos Elevados & $4,17 \%$ & $0 \%$ \\
\hline Glicose Elevada & $0 \%$ & $3,70 \%$ \\
\hline Gastrite & $4,17 \%$ & $0 \%$ \\
\hline Hipertenssão e Doenç̧as Cardiacas & $4,17 \%$ & $0 \%$ \\
\hline Hipertensão e Colesterol Elevado & $0 \%$ & $3,70 \%$ \\
\hline Hipertensão $\mathrm{e}$ Doenças Cardiacas e hipotireoidismo & $0 \%$ & $3,70 \%$ \\
\hline Bronquite & $0 \%$ & $3,70 \%$ \\
\hline
\end{tabular}

Em relação aos fatores prejudiciais a saúde, pode-se verificar que entre as mulheres o índice de sedentários encontrado é maior em comparação aos homens e o hábito de praticar atividades físicas é maior entre eles (Gráfico1), relataram jogar futebol uma vez por semana, andar de bicicleta todos os dias, praticar artes marciais três vezes por semana. Foi perguntado aos funcionários se fazem o consumo de bebidas alcoólicas e o maior consumo encontrado foi nos homens (Gráfico 2). Quanto ao hábito de fumar, a diferença entre homens e mulheres foi insignificante, podendo se observar que a maioria dos funcionários não fuma (Gráfico 3). 

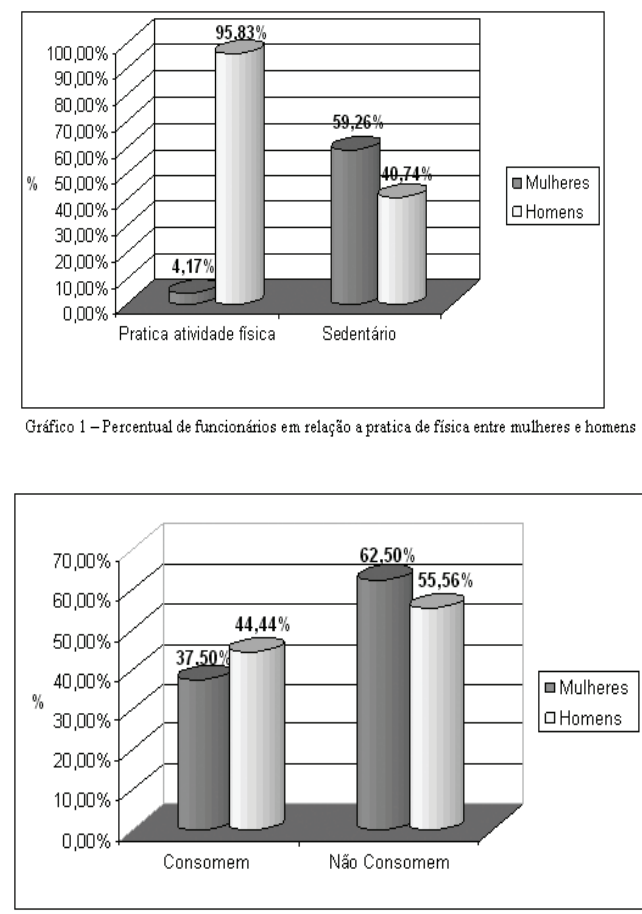

Gráfico 2 - Percentual de

Sobre seus hábitos alimentares, os funcionários foram apenas avaliados em relação ao consumo de verduras e legumes, consumidos nas refeições realizadas na unidade, a maioria, tanto homens quanto mulheres relataram consumir, sempre que oferecido, verduras e legumes, como pode-se observar no Gráfico 4. Mas relataram dar preferência a saladas como maionese e salpicão, e não gostam muito de consumir verduras, apenas consomem quando não têm outra opção no cardápio. Perguntou-se também se, na opinião do próprio funcionário, sua alimentação encontrava-se adequada, é possível notar que a maioria das mulheres não considera a própria alimentação adequada, já entre os homens observa-se o oposto, que a maioria considera a própria alimentação adequada (Gráfico 5).

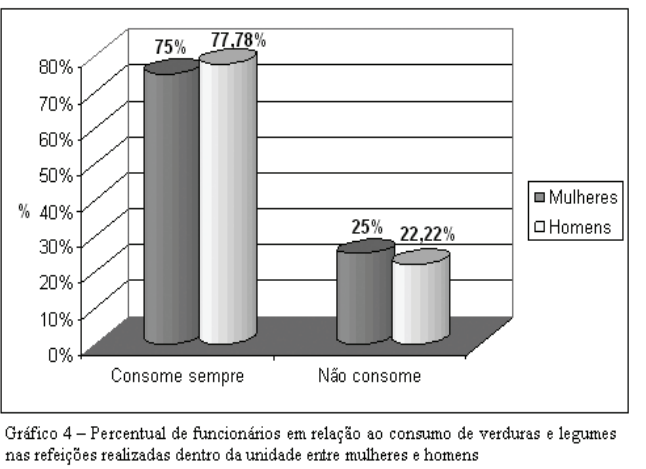

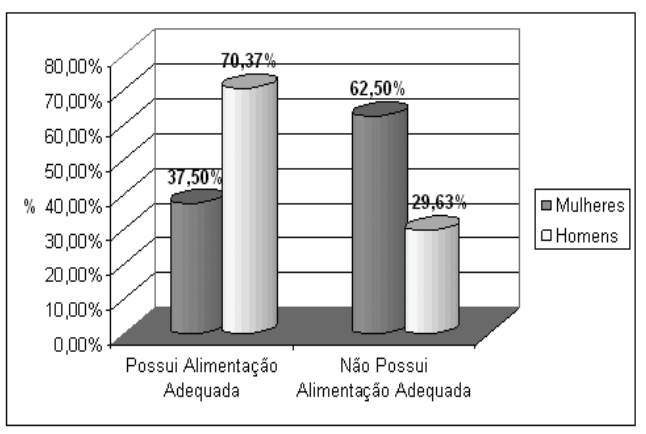

Gráfico 5 -Percentual de funcionários que consideram ou não sua alimentação adequada

Quando entregue a eles o panfleto, muitos deles alegaram não colocar em prática as informações nele contidas, mesmo considerando ter uma boa alimentação.

\section{Discussão e Conclusão}

Os dados antropométricos, obtidos neste trabalho, indicaram alta frequência de sobrepeso, (IMC $\geq$ $25 \mathrm{~kg} / \mathrm{m}^{2}$ ) o que refletiu o quadro atual encontrado em pesquisas realizadas no Brasil: o mesmo resultado encontrado por Castro (2004) ao realizar seu trabalho com operários de uma empresa metalúrgica do Rio de Janeiro. Segundo Castro (2001), os dados obtidos na Pesquisa sobre Padrões de Vida (PPV) indicaram prevalência de sobrepeso e obesidade no sexo masculino da área urbana da região Sudeste do Brasil de $37,2 \%$ e $8,0 \%$, respectivamente, sendo valor de sobrepeso bem próximos ao verificados entre os funcionários do sexo masculino.

Embora valores de IMC acima de $30 \mathrm{~kg} / \mathrm{m}^{2}$ sirvam como critério para definir obesidade em populações, seu emprego deve ser cauteloso, pois esse índice, na verdade, não mede o excesso de massa gorda. Entretanto, motivos suficientes são apresentados pelo IMC para ser utilizado em pesquisas epidemiológicas como indicador do estado nutricional (CASTRO, 2004). Por maior que seja a possível superestimativa da prevalência de sobrepeso, um grupo de funcionários em que mais da metade deles apresenta algum grau de excesso de peso já indicaria a necessidade de 
intervenção nutricional, quando os trabalhadores pudessem ser orientados individualmente.

Gomes et al. (2001) encontraram prevalência do sedentarismo no lazer de 58,9\% em homens e $77,8 \%$ em mulheres, assim como no presente estudo, que o maior índice de sedentarismo encontrado foi entre as mulheres $95,83 \%$ e homens com $40,74 \%$ sendo menos da metade.

Foi possível verificar que o consumo de verduras e legumes é maior entre as mulheres, mesmo resultado observado por Figueiredo et al. (2008) em seu estudo realizado com adultos da cidade de São Paulo.

De acordo com Jomori (2008) a escolha alimentar do consumidor é direcionada pela sua expectativa, ou seja, pelo valor dado ao alimento ou, por outro lado, pelo grau de liberdade dado ao indivíduo para realizar essas escolhas, conferindo a possibilidade de diferentes estilos de vida relatados no contexto alimentar. Diversos funcionários relataram ter os mesmos motivos ao escolher os alimentos que montaram seu prato. Por isso, muitos deles, a maioria mulheres, não têm uma boa alimentação.

A informação sobre o estado nutricional de um indivíduo ou de uma coletividade torna-se mais um elemento do diagnóstico, um subsídio para o planejamento de ações com a finalidade de promover a saúde (CASTRO, 2004). É importante que ocorra o acompanhamento nutricional dos funcionários, de forma individual, para que estes recebam orientações corretas em relação ao seu estado nutricional, não apenas por panfleto. Ao decorrer do estudo, foi possível verificar que muitos deles não sabiam realizar uma alimentação equilibrada e com todos os nutrientes necessários para manter o bom funcionamento do organismo. As mulheres mostraramse mais cientes ao que diz respeito a uma boa alimentação, por isso, quando questionadas, se faziam uma alimentação adequada, a maioria relatou não fazer.

Após a conclusão do trabalho com os funcionários, os mesmos foram orientados de que fazer uma alimentação equilibrada é muito importante, pois, evita doenças crônicas não transmissíveis, porém, essa alimentação deve vir acompanhada de bons hábitos e de um estilo de vida saudável.

\section{Referências Bibliográficas}

- $\quad$ ANJOS, L.A. Índice de massa corporal (massa corporal.estatura ${ }^{2}$ ) como indicador do estado nutricional de adultos: revisão da literatura. Rev. Saúde Pública. São Paulo. vol.26. no.6. Dez 1992.

- $\quad$ CASTRO,M.B.T;ANJOS,L.A; LOURENÇO,P.M. Padrão dietético e estado nutricional de operários de uma empresa metalúrgica do Rio de Janeiro, Brasil. Cad. Saúde Pública. Rio de Janeiro. vol.20. no.4. Jul./Ago. 2004.

- $\quad$ FIGUEIRE D O, I . C . R ; JAIME,P.C; MONTEIRO,C.A. Fatores associados ao consumo de frutas, legumes e verduras em adultos da cidade de São Paulo. Rev. Saúde Pública. São Paulo. 2008 ahead of print.

- $\quad$ GOMES,V.B; SIQUEIRA,K.S; SICHIERI,R. Atividade física em uma amostra probabilística da população do Município do Rio de Janeiro. Cad Saúde Pública. Rio de Janeiro. vol.17 no.4. Jul/Ago 2001.

- J O M O R I , M . M ; PROENÇA,R.P.C; CALVO,M.C.M. Determinantes de escolha alimentar. Rev. Nutrição. Campinas. vol.21. no.1. Jan./Fev. 2008.

- $\quad$ MATOS,C.H. Condições de trabalho e estado nutricional de 
operadores do setor de alimentação coletiva: um estudo de caso. Dissertação (Mestrado em Engenharia de Produção) - Programa de PósGraduação em Engenharia de Produção/ Ergonomia, Universidade Federal de Santa Catarina (UFSC), Florianópolis 2000.

- $\quad \mathrm{M} O \mathrm{~N}$ T E I R O, J . C ; SANTANA,A.M.C; DUARTE,M.F.S. et al. Análise de posturas no trabalho para entender a performance Física do trabalhador do setor de carnes do restaurante universitário da UFSC. Anais do $4^{\circ}$ Congresso Latino Americano de Ergonomia e $8^{\circ}$ Congresso Brasileiro de Ergonomia. Florianópolis (SC), p.400-406, 1997.

- NEUTZLING,M.B; et al. Freqüência de consumo de dietas ricas em gordura e pobres em fibra entre adolescentes. Rev. Saúde Pública. São Paulo. vol.41. no.3. Jun 2007.

- $\quad$ PROENÇA,R.P.C. Aspectos organizacionais e inovação tecnológica em processos de transferência de tecnologia: uma abordagem antropotecnológica no setor de Alimentação Coletiva. Tese (Doutorado em Engenharia). Programa de Pós-Graduação em Engenharia de Produção, Universidade Federal de Santa Catarina (UFSC), Florianópolis, 1996.

tecnológicanaproduçãodealimentãão coletiva. Florianópolis: INSULAR , 1997.

- $\quad$ S A N T' A N A, H . M . P ; AZEREDO,R.M.C; CASTRO,J.R. Estudo ergonômico em serviços de alimentação. Saúde em debate, Rio de Janeiro, no.42. mar 1994.

- SILVEIRA, E.A; et al. Validação do peso e altura referidos para o diagnóstico do estado nutricional em uma população de adultos no Sul do Brasil. Cad. Saúde Pública. Rio de Janeiro. vol.21. no.1. Jan./Fev. 2005.

- $\quad$ VELOSO,I.S; SANTANA,V.S; NELSON,F.O. Programas de alimentação para o trabalhador e seu impacto sobre ganho de peso e sobrepeso. Rev. Saúde Pública. São Paulo. vol.41. no.5. Out 2007.

- WORLD

HEALTH

ORGANIZATION. Obesity preventy and managing the global Epidemic. Geneve: WHO, 1997.
Endereço para Correspondência:

Acadêmica de Nutrição Fernanda de Almeida Escobar (Ciências da Saúde - UniFOA) -

Email: nandaescobar@yahoo.com.br

Centro Universitário de Volta Redonda

Campus Três Poços

Av. Paulo Erlei Alves Abrantes, $n^{\circ} 1325$, Três Poços - Volta Redonda / RJ CEP: 27240-560 
Anexo 1 - Panfleto (frente e verso) entregue aos funcionários da unidade.

$\rightarrow$ Tomar bastante água, porém não tomar líquido junto com as refeições:

$\rightarrow$ Ter uma alimentação balanceada não significa uma alimentação cara e sim uma alimentação simples, preparada com pouco açúcar e pouco sal, sendo conservados adequadamente e preparados de forma higiênnica.

QUAIS AS VANTAGENS DE SE TER UMA BOA ALIMENTACÃO???

$\rightarrow$ Nos fornece saúde, capacidade de trabalhar, estudar, divertir-se, etc.

$\rightarrow$ Dela depende o nosso bem estar físico e mental.

COMO CONSEGUIMOS UMA ALIMENTAÇÃO EQUILIBRADA???

$\rightarrow$ É tendo no prato arroz, feijão, carne (branca ou vermelha), salada e legumes diariamente.

IMPORTANTE: Tanto o excesso quanto a falta de alimento geram doenças, sendo necessário que tenhamos uma alimentação correta para poder prevenir doencas como: desnutrição, hipertensão, obesidade, diabetes, doenças do coração, etc.

\section{CARBOIDRATOS "ALIMENTOS} ENERGÉTICOS"

Esse grupo de alimentos são os principais fornecedores de energia, para realização de atividades diárias. Suas principais de atividades diárias. Suas principals
fontes são: pães, batatas, macarrão, biscoitos, farinhas, milho...

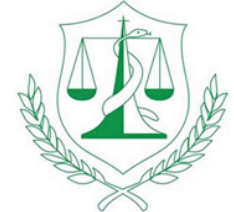

\section{ALIMENTAÇÃO ADEQUADA}

$=$

GARANTIA DE SAÚDE E BEMESTAR

\section{INFORMATIVO REALIZADO POR: \\ FERNANDA DE ALMEIDA ESCOBAR AC ADÊMICA DO UNIFOA 200520351}

\section{SOB SUPERVISÃO DE ESTÁGIO:}

AURIENE PINHEIRO DE MORAES NUTRICIONIST $A$ CRN4 - 07100277

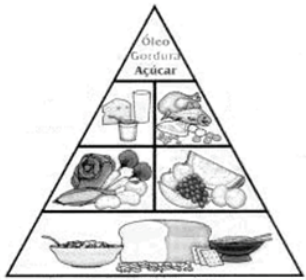

VITAMINAS, MINERAIS E FIBRAS "ALIMENTOS REGULADORES E PROTETORES"

Esse grupo de alimentos deve estar presente, pelo menos em duas refeições diárias, para que sejam alcançadas as necessidades recomendadas

5ão responsáveis pelo funcionamento perfeito do organismo. É o verdadeiro sina verde para a saúde.

Suas principais fontes são: frutas, verduras e legumes.

\section{PROTEÍNA "ALIMENTOS CONSTRUTOPES"}

Esses alimentos devem ser consumidos pelo menos quatro vezes por dia (na forma de leite $e$ derivados e carnes), pois além de atuarem no tratamento da osteoporose, construção de tecidos.

Suas fontes alimentares são: carnes, ovos, leite e derivados, feijão, soja e grãos..

"ALIMENTOS DE BAIXO TEOR NUTRITIVOS E ALTO VALOR CALÓRICO"

Esses alimentos se consumidos em excesso podem causar obesidade, diabetes mellitus, dislipidemia e doenças do coraç̃o.
Suas fontes são: óleos, açúcares, frituras e alimentos industrializados.

\section{RECOMEND AÇÕES NUTRICIONAIS} GERAIS

$\rightarrow$ Comer com calma, mastigar bem os alimentos, mantendo horário para as refeições:

$\rightarrow$ Fazer mais refeicões por dia em pequenas quantidades (café da manhã, lanche, almoço, lanche, jantar, lanche):

$\rightarrow$ Diminuir no possivel o consumo de gorduras animais, substituindo pelo consumo moderado das gorduras vegetais:

$\rightarrow$ Recomenda-se os alimentos desnatados:

$\rightarrow$ Alimentos cozidos, grelhados, refogados alimentos fritos:

$\rightarrow$ Evitar ao máximo alimentos industrializados:

$\rightarrow$ Controlar o consumo de sal e açúcar:

$\rightarrow$ Não abusar no uso do café e chá preto diariamente, devem ser consumidos com moderação, dando preferência aos chás caseiros. 